05,13

\title{
Конкуренция доменных стенок и фазы обратной намагниченности в магнитной релаксации спинового переключателя $\mathrm{Pt} / \mathrm{Co} / \mathrm{Ir} / \mathrm{Co} / \mathrm{Pt}$
}

\author{
(C) Р.Б. Моргунов ${ }^{1,2}$, Г.Л. Львова ${ }^{1,2}$, A. Hamadeh ${ }^{3}$, S. Mangin ${ }^{3}$ \\ ${ }^{1}$ Институт проблем химической фризики РАН, \\ Черноголовка, Россия \\ ${ }^{2}$ Тамбовский государственный технический университет, \\ Тамбов, Россия \\ ${ }^{3}$ Institut Jean Lamour, \\ Université de Lorraine, France \\ E-mail: morgunov2005@yandex.ru \\ (Поступила в Редакцию 26 апреля 2017 г. \\ В окончательной редакции 17 мая 2017 г.)
}

\begin{abstract}
В многослойной гетероструктуре $\mathrm{Pt} / \mathrm{Co} / \mathrm{Ir} / \mathrm{Co} / \mathrm{Pt} / \mathrm{GaAs}$ наблюдается длительная (до нескольких часов) магнитная релаксация между двумя стабильными состояниями намагниченности системы. Перемагничивание слоев гетерострукуры происходит как за счет образования зародышей доменов обратной намагничеснности, так и в результате их дальнейшего роста посредством движения доменных стенок. Конкуренция между этими двумя процессами обуславливает неэкспоненциальный характер магнитной релаксации. При $300 \mathrm{~K}$ вклады этих процессов в релаксацию намагниченности сопоставимы, в то время как при температурах ниже $200 \mathrm{~K}$ вклад зародышеобразования подавляется и магнитная релаксация происходит в результате движения доменных стенок.
\end{abstract}

Работа выполнена при поддержке гранта 3.1992.2017/ПЧ в рамках конкурса научных проектов, выполняемых научными коллективами исследовательских центров и (или) научных лабораторий образовательных организаций высшего образования.

DOI: 10.21883/FTT.2018.01.45291.143

\section{1. Введение}

В настоящее время многослойные структуры используются в устройствах спинтроники, для которых важны тонкие пленки с высоким значением перпендикулярной магнитной анизотропии, обеспечивающей гигантское магнитосопротивление [1]. В многослойных системах, состоящих из слоев Со, разделенных „немагнитными“ прослойками $\mathrm{Pt}, \mathrm{Pd}, \mathrm{Ni}, \mathrm{Au}$ и $\mathrm{Ag}$, варьирование толщины ферромагнитных слоев позволяет изменять намагниченность насыщения и значения констант магнитной анизотропии. При толщине слоя Со меньше критической $(\sim 1 \mathrm{~nm})$ доминирует магнитная анизотропия интерфейса, которая создает перпендикулярную намагниченность [2-4]. Известно, что в результате напыления Со на гранулированную поверхность $\mathrm{Pt}$, происходит диффузионное смешивание $\mathrm{Co}$ и $\mathrm{Pt}$, которое приближает свойства многослойной структуры к свойствам тонких пленок сплава CoPt. В тонких пленках CoPt наблюдается одноосная симметрия и перпендикулярная магнитная анизотропия [5], в отличие от объемных сплавов $\mathrm{CoPt}$, где имеется несколько кристаллографически эквивалентных направлений [111]. Поле анизотропии достигает $12 \mathrm{~T}$ для объемных образцов $\mathrm{CoPt}$, что соответствует коэрцитивной силе $\sim 10 \mathrm{kOe}$, в то время, как в тонких пленках CoPt коэрцитивная сила составляет 0.2-0.5 kOe. Это делает тонкие пленки CoPt и диффузионные интерфейсы $\mathrm{Co} / \mathrm{Pt}$ пригодными для целей спинтроники, поскольку кроме перпендикулярной намагниченности фер- ромагнитных слоев требуется, чтобы поле, необходимое для переключения состояний намагниченности гетероструктуры, было сравнительно невысоким. Этим и был обусловлен выбор слоев $\mathrm{Pt} / \mathrm{Co} / \mathrm{Ir} / \mathrm{Co} / \mathrm{Pt}$ в нашей работе.

Одной из моделей, описывающих перемагничивание пленок и мультислоев с перпендикулярной намагниченностью, является модель „макроспина“, рассматривающая магнитный момент макроскопического слоя и взаимодействие пары слоев в рамках Гейзенберговского формализма, применимого к отдельным спинам. В небольших образцах размером $\sim 10-100 \mathrm{~nm}$, используемых для создания ячеек магнитной памяти, зарождение доменов не происходит, так как доменные стенки энергетически невыгодны. Для таких образцов, где каждый ферромагнитный слой имеет однородную намагниченность, а разворот вектора намагниченности во внешнем магнитном поле происходит в течение $\sim 0.1 \mathrm{~ns}$, применима модель „макроспина“ $[6,7]$. Однако, гетероструктуры с гигантским магнитосопротивлением применяются также и для создания сенсоров, чувствительных к присутствию ферромагнитных наночастиц на их поверхности для биомедицинских применений $[8,9]$. В этих устройствах, требующих как можно большей поверхности сенсора для размещения анализируемых препаратов в технологиях lab-on-chip, размеры устройства составляют $\sim 1 \mathrm{~mm}$. В таких образцах намагниченность пленок становится неоднородной, появляется доменная структура, а в процессе перемагничивания наблюдается длительная релаксация намагниченности. 


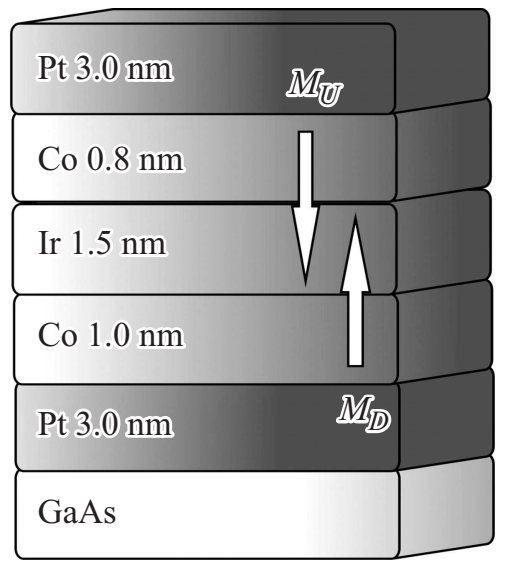

Рис. 1. Схема гетероструктуры $\mathrm{Pt} / \mathrm{Co} / \mathrm{Ir} / \mathrm{Co} / \mathrm{Pt} / \mathrm{GaAs}$. Стрелки соответствуют направлениям намагниченности $\mathbf{M}_{\mathbf{U}}$ и $\mathbf{M}_{\mathbf{D}}$ тонкого и толстого ферромагнитных слоев соответственно.

Теория „макроспина“ в такой ситуации неприменима, а динамика перемагничивания сенсоров определяется движением доменных стенок и зарождением доменов обратной намагниченности. Поэтому цель данной работы заключается в определении вкладов фазы обратной намагниченности и доменных стенок в перемагничивание спиновых переключателей $\mathrm{Pt} / \mathrm{Co} / \mathrm{Ir} / \mathrm{Co} / \mathrm{Pt}$ с размерами до нескольких миллиметров.

\section{2. Методика}

Подробная карта переключения исследуемого спинового переключателя $\mathrm{Pt} / \mathrm{Co} / \mathrm{Ir} / \mathrm{Co} / \mathrm{Pt}$ в координатах магнитное поле $H$-температура $T$ была получена в [10]. Многослойная структура $\mathrm{Pt}(3 \mathrm{~nm}) / \mathrm{Co}(0.8 \mathrm{~nm}) /$ $\operatorname{Ir}(1.5 \mathrm{~nm}) / \mathrm{Co}(1.0 \mathrm{~nm}) / \mathrm{Pt}(3 \mathrm{~nm})$ была нанесена на нелегированную подложку GaAs (001) методом магнетронного напыления в вакуумной системе при давлении около $1 \sim 10$ Torr. Слои наносились на подложку на охлаждаемом вращающемся столике. Последовательность напыленных слоев Со и $\mathrm{Pt}$ образовывала искусственную сверхрешетку с перпендикулярной магнитокристаллической анизотропией (рис. 1). Кратковременный отжиг в течение $3 \mathrm{~min}$ проводили при $250^{\circ} \mathrm{C}$ для кристаллизации слоев $\mathrm{Pt} / \mathrm{Co}$ и увеличения значения перпендикулярной магнитной анизотропии. Во время этого процесса диффузионный слой Со смешивался с промежуточным слоем Ir, что усиливало антиферромагнитное взаимодействие между двумя соседними слоями Со. Переходный металл Ir был выбран в качестве промежуточного немагнитного слоя потому, что, во-первых, он обеспечивает высокое значение гигантского магнитосопротивления, превышающего $65 \%$ в структурах $\mathrm{Cu} / \mathrm{Co}[5,6]$, а, во-вторых, простая модель свободных электронов правильно предсказывает применимость теории RKKY для описания осциллирующего поведения обменного взаимодействия в зависимости от толщины слоя Ir [11].
Петли магнитного гистерезиса в диапазоне $150-300 \mathrm{~K}$ и временные зависимости намагниченности при различных температурах были получены для образцов в форме пластинки размерами $0.4 \times 4 \times 5 \mathrm{~mm}$ с помощью магнитометра MPMS 5XL Quantum Design.

\section{3. Экспериментальные результаты}

Петли магнитного гистерезиса для двухслойной системы были записаны в диапазоне температур $150-300 \mathrm{~K}$. На рис. 2 показаны примеры типичных петель гистерезиса при 200 и $300 \mathrm{~K}$. На рис. 2 изображены намагниченности ферромагнитных слоев гетероструктуры для ее четырех стабильных состояний $M_{1}, M_{2}, M_{3}, M_{4}$. Правая стрелка соответствует намагничиванию „толстого“ слоя $1 \mathrm{~nm}$. Левая стрелка соответствует намагничиванию „тонкого“ слоя $0.8 \mathrm{~nm}$. Состояния $M_{1}$ и $M_{4}$ соответствуют параллельным ориентациям намагниченности $(\uparrow \uparrow, \downarrow \downarrow)$ верхнего и нижнего слоя. Магнитные
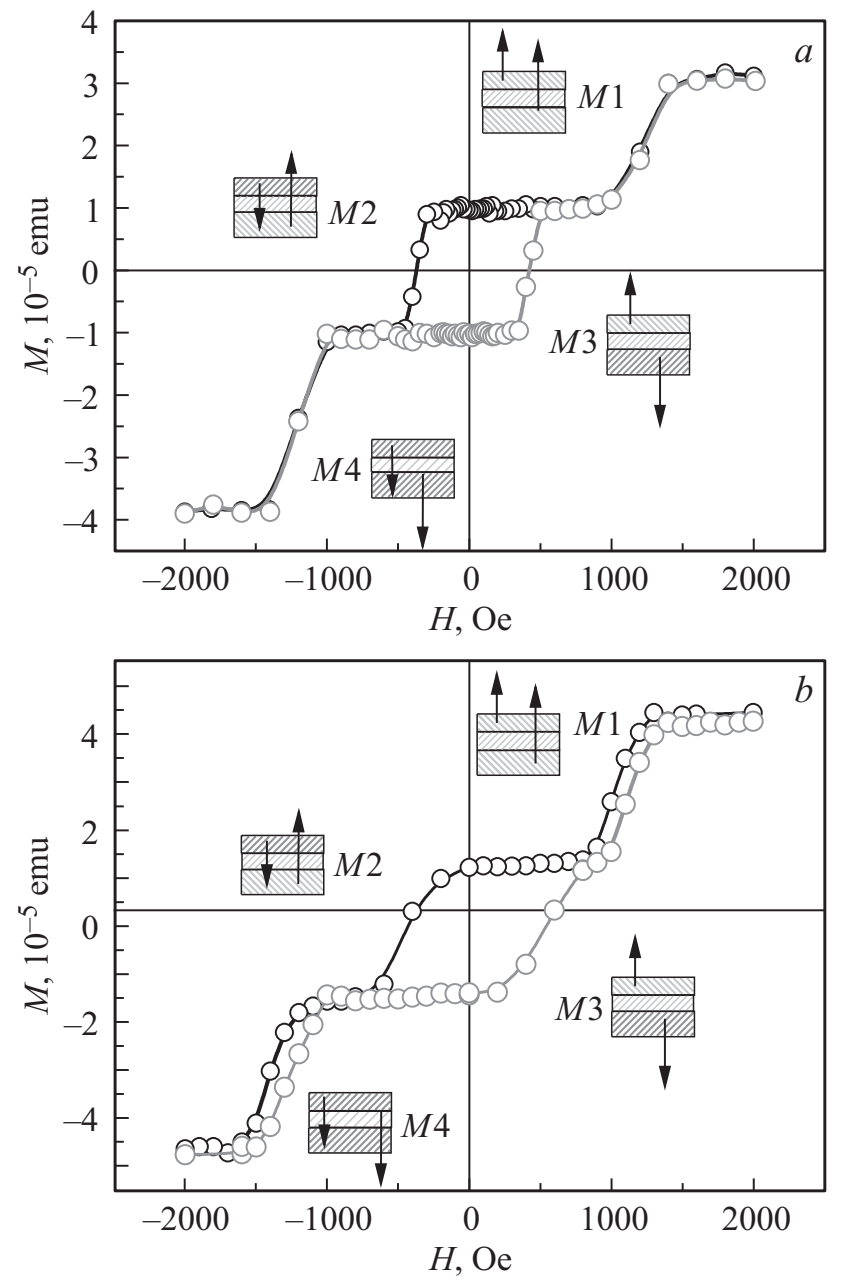

Рис. 2. Гистерезисы намагниченности при $T=300 \mathrm{~K}(a)$ и $T=200 \mathrm{~K}(b)$. Символы $M_{1}, M_{2}, M_{3}, M_{4}$ соответствуют стабильным состояниям намагниченности гетероструктуры, показанным на врезках. Стрелки соответствуют направлениям намагниченности $\mathbf{M}_{\mathbf{U}}$ и $\mathbf{M}_{\mathbf{D}}$ толстого и тонкого слоя $\mathrm{Co}$. 

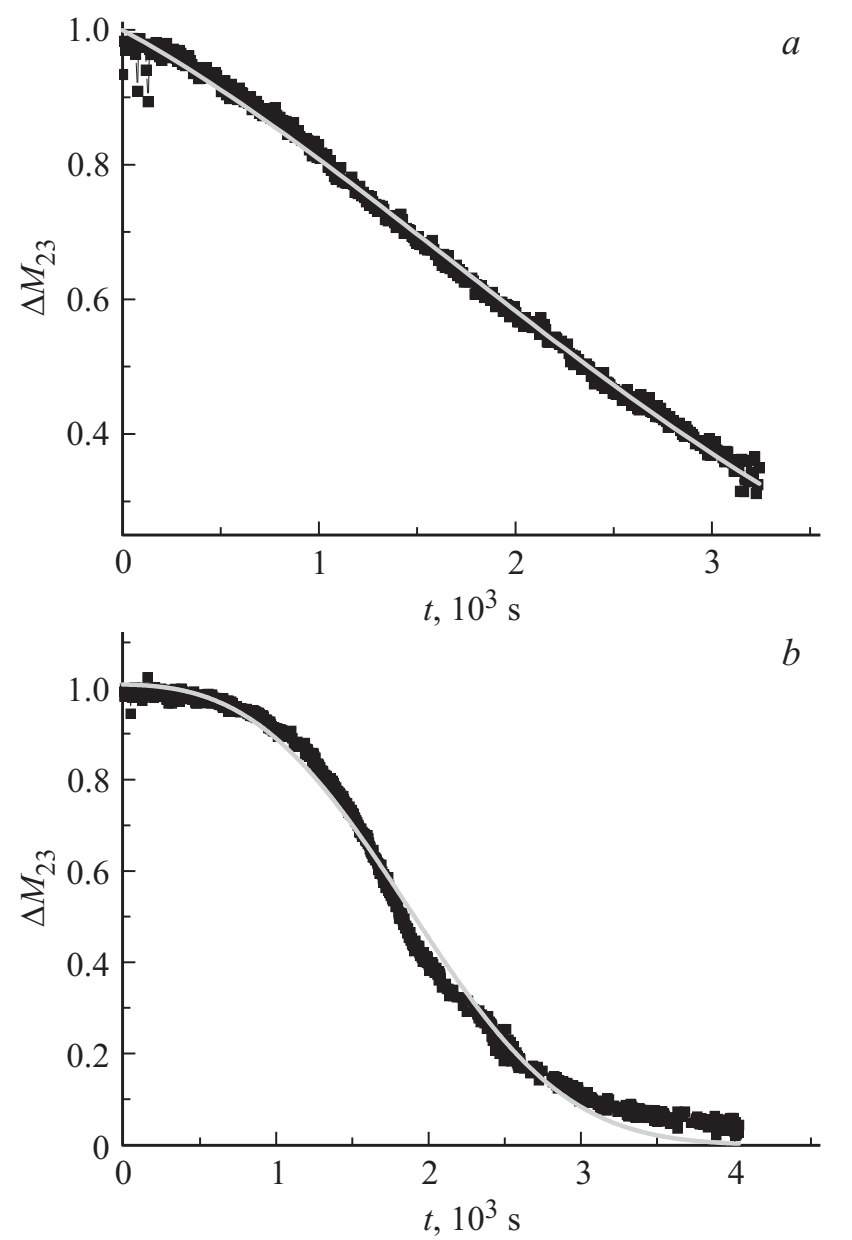

Рис. 3. Зависимости относительной намагниченности $\Delta M_{23}=\left(M(t)-M_{3}\right) /\left(M_{2}-M_{3}\right)$ от времени при $300 \mathrm{~K}(a)$ и $200 \mathrm{~K}(b)$. Аппроксимации моделью Фаттузо-Лабруне, описанной в тексте, показаны сплошными линиями.

моменты $\mathbf{M}_{\mathbf{U}}$ и $\mathbf{M}_{\mathbf{D}}$ этих слоев направлены вдоль $\uparrow \uparrow$ или против $\downarrow \downarrow$ направления приложенного поля $H$. Состояния $M_{2}, M_{3}$ соответствуют антипараллельным взаимным ориентациям $\mathbf{M}_{\mathbf{U}}$ и $\mathbf{M}_{\mathbf{D}}$, которые различаются направлением суммарного магнитного момента по отношению к внешнему магнитному полю, так как $M_{\mathrm{U}} \neq M_{\mathrm{D}}$.

Магнитные петли гистерезиса, записанные в диапазоне температур 150-300 K, представлены на рис. 2, $a, b$. Магнитная петля гистерезиса содержит эквивалентные переходы $M_{1} \leftrightarrow M_{2}$ и $M_{3} \leftrightarrow M_{4}$, соответствующие развороту вектора намагниченности тонкого слоя (рис. 2,a). Середины этих переходов соответствуют магнитному полю $\pm 1.2 \mathrm{kOе}$. Другой переход $M_{2} \leftrightarrow M_{3}$ соответствует одновременному развороту векторов намагниченности тонкого и толстого слоев. Середина этого перехода соответствует магнитному полю 420 Ое. Остальные переходы между состояниями намагниченности гетероструктуры при этой температуре не реализуются.

Для выявления механизмов перемагничивания ферромагнитных слоев были измерены временные зависимости релаксации магнитного момента $M(t)$ при перехо- де $M_{2} \leftrightarrow M_{3}$. Образец намагничивался полем $10 \mathrm{kOe}$, перпендикулярным пленке, в течение $300 \mathrm{~s}$. Затем поле переключали к новому значению -350 Ое и записывали временную зависимость $M(t)$. Длительность переключения магнитного поля („мертвого времени“) была значительно меньше длительности магнитной релаксации и составляла $60 \mathrm{~s}$. На рис. 3 показаны зависимости величин относительной намагниченности $\Delta M_{23}=\left(M(t)-M_{3}\right) /\left(M_{2}-M_{3}\right)$ от времени при $300 \mathrm{~K}$ (рис. $3, a)$ и $200 \mathrm{~K}$ (рис. 3, b). При изменении температуры от $300 \mathrm{~K}$ (рис. $3, a$ ) к $200 \mathrm{~K}$ (рис. $3, b$ ) форма кривой магнитной релаксации качественно изменяется, так как на ней появляется перегиб. Это связано с изменением механизмов магнитной релаксации.

\section{4. Обсуждение}

Как правило, термоактивированное перемагничивание ферромагнетиков определяется зародышеобразованием фазы обратной намагниченности [12] и/или движением доменных стенок [13]. Оба эти процесса учтены в модели Фаттузо-Лабруне [14], описывающей релаксацию намагниченности тонких пленок. В рамках этой модели временная зависимость перемагничивания ферромагнитной пленки может быть описана формулой [14]

$$
\begin{aligned}
(M(t) & \left.+M_{\mathrm{S}}\right) / 2 M_{\mathrm{S}}=\exp \left(-k^{2}\left(2-2\left(R t+k^{-1}\right)\right.\right. \\
& \left.\left.+\left(R t+k^{-1}\right)^{2}-2 e^{-R t}\left(1-k^{-1}\right)-k^{-2}(1-R t)\right)\right),
\end{aligned}
$$

где $M_{\mathrm{S}}-$ намагниченность насыщения, $R-$ частота зародышеобразования фазы обратной намагниченности в единицу времени, $k=v / R r_{0}-$ безразмерный параметр, характеризующий конкуренцию движения доменных стенок и нуклеации фазы обратной намагниченности, и выражаемый через скорость движения доменных стенок $v$ и начальный радиус зародышей обратной намагниченности $r_{0}$. Таким образом, аппроксимация зависимости $\Delta M_{23}(t)$ позволяет определить параметры $R$ и $k$.

Примеры аппроксимации температурных зависимостей $\Delta M_{23}$ уравнением (1) представлены на рис. $3, a, b$ сплошными линиями. Полученные параметры аппроксимации показаны в таблице. При $T=300 \mathrm{~K}$ значение $k=1.9$, согласно [14], отвечает конкуренции между зарождением фазы обратной намагниченности и движением доменных стенок. При $T=200 \mathrm{~K}$, когда $k=559$, доминирующим механизмом магнитной релаксации является процесс распространения доменных стенок. При-

Параметры аппроксимации временной зависимости относительной намагниченности формулой Фаттузо-Лабруне при двух температурах

\begin{tabular}{c|c|c}
\hline Параметр & $T=200 \mathrm{~K}$ & $T=300 \mathrm{~K}$ \\
\hline$R, 10^{-5}, \mathrm{~s}^{-1}$ & $6 \pm 1$ & $16 \pm 2$ \\
$k$ & $559 \pm 10$ & $1.9 \pm 0.1$
\end{tabular}


чиной „замораживания“ вклада нуклеации доменов обратной намагниченности при понижении температуры является уменьшение вероятности их термоактивированного зарождения. Частота возникновения зародышей фазы обратной намагниченности $R$ также значительно уменьшается с понижением температуры (таблица), однако не так быстро, как это происходит для доменных стенок, поскольку эти процессы имеют разные энергии активации.

\section{5. Выводы}

В многослойной структуре $\mathrm{Pt} / \mathrm{Co} / \mathrm{Ir} / \mathrm{Co} / \mathrm{Pt} / \mathrm{GaAs}$ обнаружена длительная магнитная релаксация между стабильными состояниями системы. В рамках модели Фаттузо-Лабруне определен параметр $k$, характеризующий вклады фазы обратной намагниченности и доменных стенок в переходный процесс между стабильными состояниями намагниченности. При $T=300 \mathrm{~K}$ величина $k=1.9$ соответствует приблизительно одинаковому вкладу зарождения фазы обратной намагниченности и движения доменных стенок в магнитную релаксацию. При $T=200 \mathrm{~K}$, когда $k=559$, основной причиной магнитной релаксации является распространение доменных стенок.

\section{Список литературы}

[1] I. Zǔtic, J. Fabian, S. Das Sarma. Rev. Mod. Phys. 76, 323 (2004).

[2] S. Bandiera, R.C. Sousa, B. Rodmacq, B. Dieny. IEEE Magn. Lett. 2, 3000504 (2011).

[3] N. Honda, S. Hinata, S. Saito. AIP Adv. 7, 056518 (2017).

[4] F.J.A. den Broeder, D. Kuiper, A.P. van de Mosselaer, W. Hoving. Phys. Rev. Lett. 60, 2769 (1988).

[5] C.J. Lin, G.L. Gorman. Appl. Phys. Lett. 61, 1600 (1992).

[6] J. Xiao, A. Zangwill. M.D. Stiles. Phys. Rev. B 72, 014446 (2005).

[7] F. Wegelin, D. Valdaitsev, A. Krasyuk, S.A. Nepijko, G. Schönhense, H.J. Elmers, I. Krug, C.M. Schneider. Phys. Rev. B 76, 134410 (2007).

[8] H.A. Ferreira, D.L. Graham, P.P. Freitas, J.M.S. Cabral. J. Appl. Phys. 93, 7281 (2003).

[9] G. Li, S. Sun, R.J. Wilson, R.L. White, N. Pourmand, S.X. Wang. Sens. Actuators A 126, 98 (2006).

[10] R. Morgunov, A. Hamadeh, T. Fache, G. Lvova, O. Koplak, A. Talantsev, S. Mangin. Superlattices, Microstructures 104, 509 (2017).

[11] Y. Luo, M. Moske, K. Samwer. Europhys. Lett. 42, 5, 565 (1998).

[12] M. Labrune, S. Andrieu, F. Rio, P. Bernstein. J. Magn. Magn. Mater. 80, 211 (1989).

[13] E. Fatuzzo. Phys. Rev. 127, 1999 (1962).

[14] A.A. Adjanoh, R. Belhi, J. Vogel, M. Ayadi, K. Abdelmoula. J. Magn. Magn. Mater. 323, 504 (2011). 